\title{
The development of a real-time PCR assay for the detection of azole resistance in Aspergillus fumigatus positive patients
}

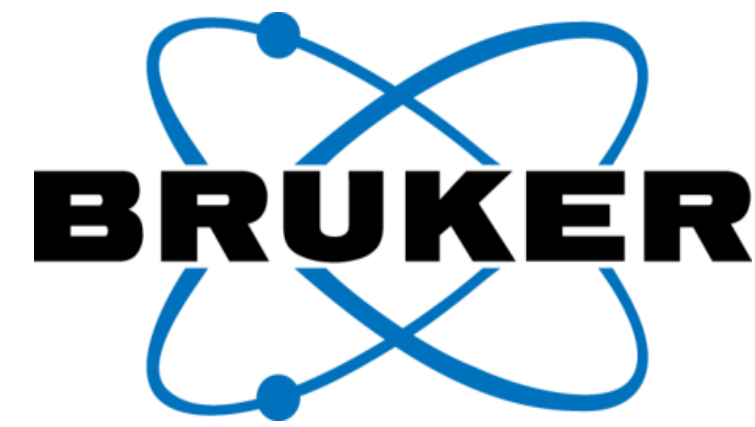

Selina Henriquez, Kate Dempsey, Colette Dalton, Tara Donnelly \& Julie Green* Bruker Microbiology, Glasgow, UK julie.green@bruker.com

\section{Introduction}

Invasive aspergillosis (IA) is associated with high mortality and morbidity in immunosuppressed patients. IA is difficult to diagnose and is often combatted by the use of prophylactic or empiric anti-fungal therapy, primarily using the azole class of drugs.

Azole resistance in A. fumigatus, as a result of mutations in the Cyp51A gene, is an emerging clinical problem ${ }^{1}$ (Figure 1). The 2017 guidelines for management of invasive fungal infections from the Dutch Working Party on Antibiotic Policy has recommended that PCR is used to test for Cyp51A mutations ${ }^{2}$.

Blood culture has limitations on the diagnosis of IA - slow to grow, with poor sensitivity and tending towards positivity late in disease. PCR is becoming a useful aid to the diagnosis of IA.

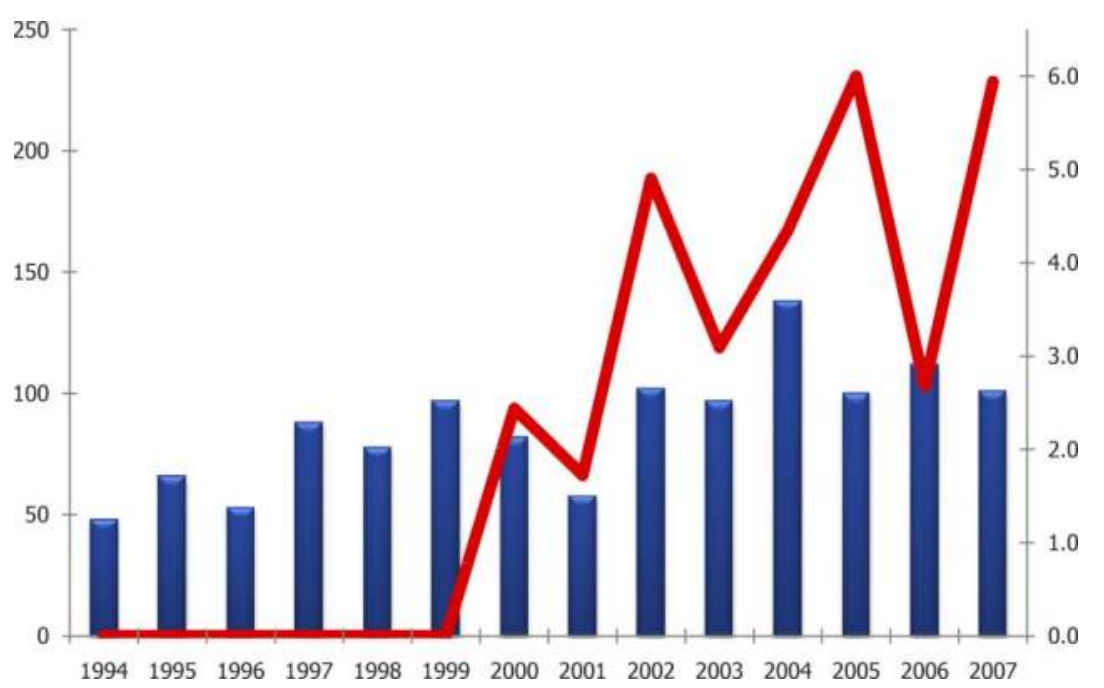

Fig 1: Epidemiology of Itraconazole Resistance in Dutch A. fumigatus isolates ${ }^{1}$ Blue bars represent the number of patients with a positive $A$. fumigatus culture (left $y$-axis) and the red line represents the percentage of those patients with an Azole resistant isolate (right $y$-axis).

Bruker have launched a CE-IVD A. fumigatus azole resistance PCR; Fungiplex ${ }^{\circledR}$ Aspergillus Azole- $\mathrm{R}$, which targets the Cyp51A gene, and differentiates the $\mathrm{TR}_{34}$ and $\mathrm{TR}_{46}$ mutations, associated with pan-azole resistance. The validation of this Kit is described herein

\section{Methods - Experimental Design}

Observation of Analytical Specificity was achieved by extracting 10 wild type strains and 11 azole resistant strains of $A$. fumigatus and testing at a concentration of 10,000 genome equivalents per PCR.

Observation of Analytical Sensitivity was determined by spiking various concentrations of genomic DNA from either $A$. fumigatus with a TR34 mutation or $A$. fumigatus with a TR46 mutation into serum, plasma and BAL. Final burdens of 10,000, 1000, 500, 250, 100, 75, 50, 25, 10, 5 and 1 genome $/ 0.5 \mathrm{~mL}$ of sample were prepared.

Clinical Performance was assessed using retrospective clinical isolates from cystic fibrosis patients.

All samples were extracted using either a Biomérieux EasyMAG or a Biomérieux Emag producing a final elution volume of $60 \mu \mathrm{L}$. All PCRs were performed using a Qiagen Rotor-Gene Q platform

For all studies Fungiplex Aspergillus Azole-R PCR was performed in duplicate alongside duplicate testing using the Fungiplex Aspergillus PCR Kit to establish a relationship between the sensitivities of the two assays.

\section{Results}

The results of the analytical specificity study are provided in Table 1 . At the high concentration tested, 10,000 ge, no cross reactivity was observed between the resistance mutation assay and the wild type strains.
Table 1: Results of the analytical specificity testing 10,000 ge per reaction

\begin{tabular}{|l|c|c|}
\hline Strains & $\begin{array}{c}\text { Detected by } \\
\text { Fungiplex } \\
\text { Aspergillus Azole-R }\end{array}$ & $\begin{array}{c}\text { Detected by } \\
\text { Fungiplex Aspergillus }\end{array}$ \\
\hline Wild type $A$. fumigatus $\times 10$ & $\times$ & $\checkmark$ \\
\hline TR34 mutation $\times 10$ & $\checkmark$ & $\checkmark$ \\
\hline TR46 mutation $\times 1$ & $\checkmark$ & $\checkmark$ \\
\hline
\end{tabular}

The limits of detection (LOD) achieved from the sensitivity study are provided in Table 2 per sample matrix used for preparation of the simulated samples. The reproducible LODs were determined from duplicate testing with positivity in at least 1 replicate considered significant. Non-reproducible detection was observed to 5 ge.

Table 2: Results of the analytical sensitivity testing to define the LODs

\begin{tabular}{|l|c|}
\hline Sample & $\begin{array}{c}\text { Limit of Detection } \\
\text { (6/6 extracts detected) }\end{array}$ \\
\hline TR34 marker in serum & $50 \mathrm{ge}$ \\
TR34 marker in plasma & $75 \mathrm{ge}$ \\
TR34 marker in BAL & $25 \mathrm{ge}$ \\
\hline TR46 marker in serum & $50 \mathrm{ge}$ \\
TR46 marker in plasma & $50 \mathrm{ge}$ \\
TR46 marker in BAL & $50 \mathrm{ge}$ \\
\hline
\end{tabular}

The results of testing the retrospective clinical isolates, previously categorised as azole-resistant $A$. fumigatus with the $\mathrm{TR}_{34}$ mutation, are provided in Table 3 . The results show the correlation between positivity for the specific Fungiplex Aspergillus Azole-R Kit and the Fungiplex Aspergillus species Kit.

Table 3: Performance of the Fungiplex Aspergillus tests with clinical isolates

\begin{tabular}{|l|l|l|l|}
\hline $\begin{array}{l}\text { TR }_{34} \text { A. fumigatus } \\
\text { Samples }\end{array}$ & $\begin{array}{c}\text { Fungiplex } \\
\text { Aspergillus } \\
\text { Azole-R } \\
\text { TR34 Result }\end{array}$ & $\begin{array}{c}\text { Fungiplex } \\
\text { Aspergillus } \\
\text { Azole-R } \\
\text { TR46 Result }\end{array}$ & $\begin{array}{c}\text { Fungiplex } \\
\text { Aspergillus } \\
\text { Result }\end{array}$ \\
\hline Sample 1 & $\begin{array}{c}\text { POSITIVE } \\
\text { Ct }=20.2\end{array}$ & NEGATIVE & $\begin{array}{r}\text { POSITIVE } \\
\text { Ct }=21.7\end{array}$ \\
\hline Sample 2 & $\begin{array}{l}\text { POSITIVE } \\
\text { Ct }=17.8\end{array}$ & NEGATIVE & $\begin{array}{c}\text { POSITIVE } \\
\text { Ct }=19.5\end{array}$ \\
\hline Sample 3 & POSTIVE & NEGATIVE & POSTIVE \\
& Ct $=20.7$ & Ct $=22.6$
\end{tabular}

\section{Conclusions}

- Fungiplex Aspergillus Azole-R detects and differentiates the two most common Cyp51A mutations associated with pan-azole resistance, $\mathbf{T R}_{\mathbf{3 4}}$ and $\mathbf{T} \mathbf{R}_{\mathbf{4 6}}$.

- Analytical specificity is $\mathbf{1 0 0} \%$ and LODs prove it is possible to detect low fungal burdens, with this single copy gene.

- Using Fungiplex Aspergillus IVD and Fungiplex Aspergillus Azole-R IVD offers the potential for early diagnosis of IA and targeted therapy.

\section{Acknowledgements}

The authors thank Dr Lewis White and Raquel Posso (University Hospital Wales, Cardiff,) for performing this study and Prof. S Bretagne (Hopital Saint-Louis, Paris), Prof. J. Meis (CanisiusWilhelmina Ziekenhuis) and Dr Wilfried Posch (Medical University of Innsbruck) for provision of additional azole-resistant $A$. fumigatus strains.

References

(1) E. Snelders et al., PLOS Medicine (2008) Nov 11;5(11):e219.

(2) B.J. Kullberg et al., SWAB Guidelines for the Management

of Invasive Fungal Infections, Aug 2017 